\title{
Disseminated anetoderma associated with antiphospholipid antibodies
}

\author{
Jorge A. Esquivel-Valerio ${ }^{1}$ (D) Jesus Alberto Cardenas-de la Garza ${ }^{1}$ (D) - Rosa I. Arvizu-Rivera ${ }^{2}$ (D) \\ Dionicio A. Galarza-Delgado ${ }^{1}$ (1)
}

Received: 3 July 2020 / Revised: 25 July 2020 / Accepted: 4 August 2020 / Published online: 10 August 2020

(C) International League of Associations for Rheumatology (ILAR) 2020

\section{Presentation}

A previously healthy 32-year-old woman was referred to the rheumatology outpatient clinic due to a disseminated dermatosis and low titer antinuclear antibodies (1:40) measured by indirect immunofluorescence.

The dermatosis had started 8 months prior as flat erythematous macules and patches on her back that posteriorly disseminated to the trunk, shoulder, and cheek, becoming skin-colored. The lesions did not cause pain, pruritus, or dysesthesia. She denied arthritis, fever, obstetric complications, and history of thrombosis. Her family history was unremarkable.

On physical examination, multiple erythematous and skincolored, sac-like protrusions and plaques of variable size were present on the trunk, face, and upper extremities; additionally, she presented flat erythematous and hyperpigmented patches (Fig. 1).

Anti-cardiolipin IgG antibodies and beta-2-glycoprotein 1 antibodies were positive, and lupus anticoagulant was negative. Skin biopsy was not performed because of the characteristic clinical presentation. Her final diagnosis was primary anetoderma associated with antiphospholipid antibodies. We initiated hydroxychloroquine and low-dose aspirin. The patient referred partial improvement of the erythematous lesions and did not present new ones at 2month follow-up.

\section{Discussion}

Anetoderma is a rare skin disorder characterized by sac-like protrusions or depressions secondary to loss of elastic fibers. Primary anetoderma presents in normal skin or after a nonspecific inflammatory process, while secondary anetoderma results due to a preceding well-defined skin disease like acne $[1,2]$.

Autoimmune diseases such as systemic lupus erythematosus and antiphospholipid syndrome as well as antiphospholipid antibodies positivity have been associated with cases of primary anetoderma like the one presented herein. The pathogenic relationship between these entities is yet to be elucidated. Hypotheses include microthromboses of dermal vessels with secondary destruction of elastic fibers and autoantibodies-mediated destruction $[1,2]$. Skin biopsy may help in atypical cases or when diagnosis is uncertain. Long-term follow-up is necessary because patients may posteriorly present manifestations of autoimmune disease $[1,3]$.

Jorge A. Esquivel-Valerio

jesquive@yahoo.com

1 Department of Rheumatology, Hospital Universitario "Dr. Jose

Eleuterio Gonzalez", Universidad Autónoma de Nuevo León, Monterrey, Nuevo Leon, Mexico

2 Department of Internal Medicine, Hospital Universitario "Dr. Jose Eleuterio Gonzalez", Universidad Autónoma de Nuevo León, Monterrey, Nuevo Leon, Mexico 

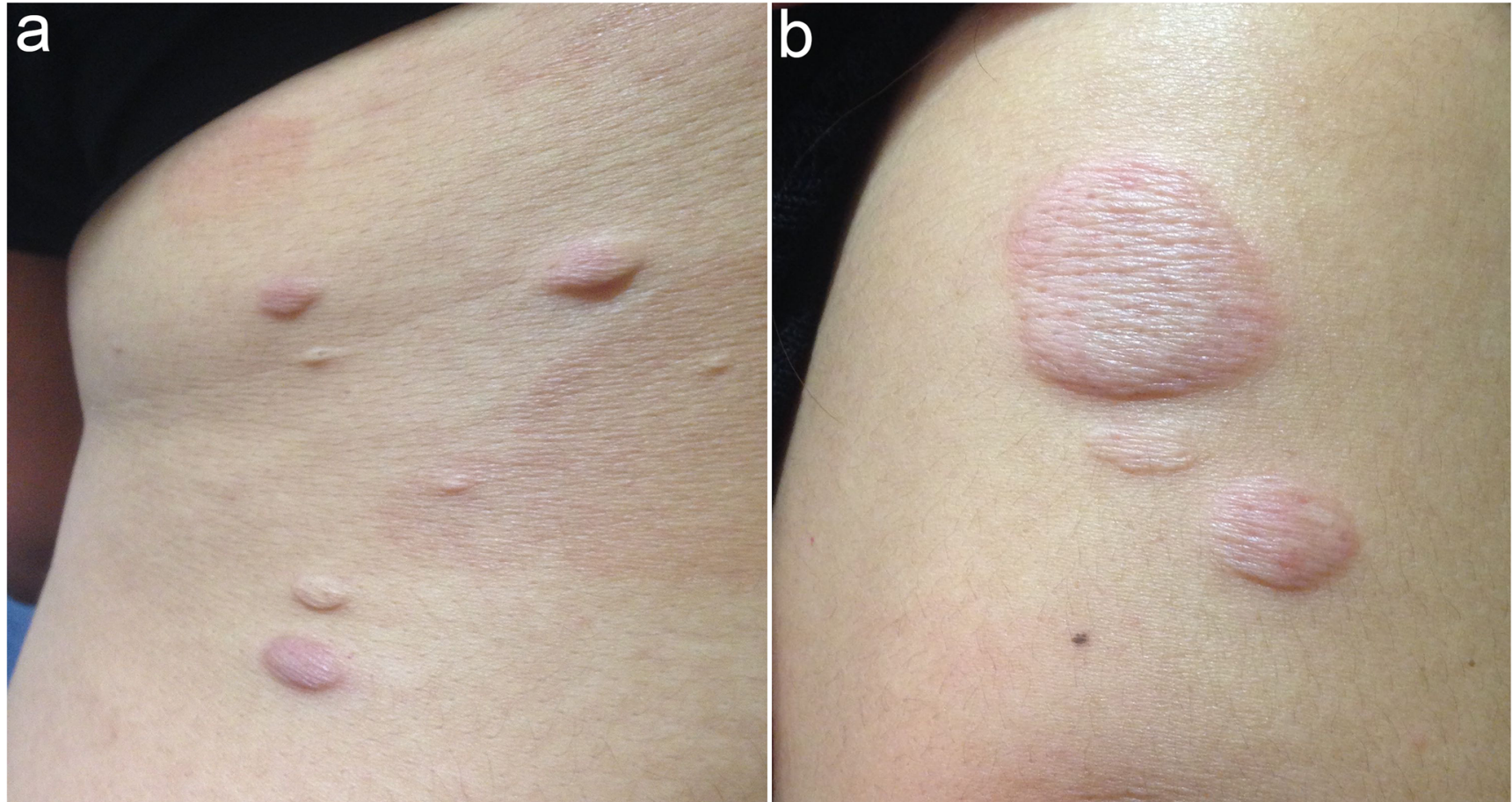

Fig. 1 Erythematous and skin-colored, sac-like protrusions and erythematous and hyperpigmented patches on the patient's back (a) and upper arm (b)

Data availability Additional information is available from the corresponding author on reasonable request.

\section{Compliance with ethical standards}

\section{Disclosures None.}

Ethics approval Ethical approval was not required because of the anonymity of the images.

Consent to participate Patient's written consent to participate and for publication was obtained.

Consent for publication Patient's written consent to participate and for publication was obtained.

Code availability We did not use any new software application or custom code.

\section{References}

1. Iglesias-Puzas Á, Batalla A, Peón G, Álvarez C, Flórez Á (2019) Anetoderma and systemic lupus erythematosus: case report and literature review. Acta Derm Venereol 99:335-336. https://doi.org/10. 2340/00015555-3100

2. Hodak E, Michael D (2007) Primary anetoderma and antiphospholipid antibodies-review of the literature. Clin Rev Allergy Immunol 32:162-166. https://doi.org/10.1007/s12016007-0011-1

3. Genta MP, Abreu MAMM, Nai GA (2020) Anetoderma: an alert for antiphospholipid antibody syndrome. An Bras Dermatol 95:123125. https://doi.org/10.1016/j.abd.2019.04.010

Publisher's note Springer Nature remains neutral with regard to jurisdictional claims in published maps and institutional affiliations. 